\begin{tabular}{|c|c|}
\hline Title & $\begin{array}{l}\text { Formation of a Structure of Exponentially Forking Branches with a Steady-state A mount of Current-year Shoots in a } \\
\text { Hardwood Tree Crown }\end{array}$ \\
\hline Author(s) & Sumida, A kihiro; Takai, Y uri \\
\hline Citation & $\begin{array}{l}\text { https://doi.org/10.1007/978-4.431-65958-721 } \\
\text { T. Sekimura, S. Noji, N. V ena, P.K. Maini (Eds.) } 『 \text { Morphogenesis and Pattern Formation in Biological Systems: } \\
\text { Experiments and Models』 (ISBN: } 978-4-431-00644 \text { 2) pp. 247-256 }\end{array}$ \\
\hline Issue Date & 2003 \\
\hline Doc URL & http:/hdl.handle.net/2115/44835 \\
\hline Rights & The original publication is available at www.springerlink.com \\
\hline Type & article (author version) \\
\hline File Information & sumida2003.pdf \\
\hline
\end{tabular}

Instructions for use 


\section{Manuscript of}

SUMIDA, A. TAKAI, Y. (2003) Formation of a structure of exponentially forking branches with a steady-state amount of current-year shoots in a hardwood tree crown.

\section{In: T. Sekimura, S. Noji, N. Ueno, P.K. Maini (Eds.)}

"Morphogenesis and Pattern Formation in Biological Systems: Experiments and Models." pp 247-256. Springer-Verlag, Tokyo, 2003.

Note; The article above contains errata $\left(^{*}\right)$, which have been revised in this manuscript.

* Line $15-16$ of the first paragraph in page 248 , "due to the strong apical dominance" should read "due to the lack of strong apical dominance"

* Eq. (21.1) in page 250, [ND] $=(\mathrm{LM}-1.09) / 2.26$, should read $\mathrm{ND}=[(\mathrm{LM}-1.09) / 2.26]$ 


\title{
Formation of a structure of exponentially forking branches with a steady-state amount of current-year shoots in a hardwood tree crown
}

\author{
AKIHIRO SUMIDA $^{1}$ AND YURI TAKAI ${ }^{2}$ \\ ${ }^{1}$ Institute of Low Temperature Science, Hokkaido University, N19W8, Sapporo \\ 060-0819, Japan. \\ asumida@lowtem.hokudai.ac.jp \\ ${ }^{2}$ Faculty of Agriculture, Gifu University
}

1 Introduction

Forking branches are a structural pattern characterizing a tree species. For a tree species as a sessile organism, the forking structure is essential for gaining solar energy by spreading leaves in lighter spaces as quickly as possible. The structure is an outcome of a process in which a mother shoot of a branch produces multiple daughter shoots, and thus allows a tree to exponentially increase the amount of leaves available for photosynthetic production. On the other hand, in closed hardwood forests, tree crowns are so close to each other that there is very little space for "exponential" expansion of tree crowns (Sumida et al. 2002). How can the nature of branch forking, which appears to result in an exponential increase of leaf amount, be consistent with crown development in closed hardwood stands where crown expansion is limited due to a lack of available space? To answer such a question, analyses of demographic (birth and death) and morphological patterns of annual shoots (the portion of shoots elongated during a year) in tree crowns are useful, since they can clarify how shoot population in a crown develops and is maintained (Steingraeber et al. 1979; White 1979; Koike 1989; Wilson 1989; Collet and Frochot 1996; Sattler and Rutishauser 1997; Chaar et al. 1997; Buck-Sorlin and Bell 2000; Suzuki 2000; Suzuki 2001; Seleznyova et al. 2002). Here, we begin by showing an example of the structural pattern of a branch as observed in the top canopy of a closed hardwood forest. We then show how the observed pattern can be formed by introducing a model simulating demographic and structural patterns of the 
annual shoots.

2 Estimation of future branch development based on a branch structure at a point in time

If the demographic pattern of annual shoots in a crown is followed over time, it would take years to complete an observation. Hence, a demographic pattern is often estimated from an intensive observation of a structural pattern of a branch at a point in time. In the case of our target species, the deciduous oak Konara (Quercus serrata Thunb.), the extension of the growth of current-year shoots is monopodial with rhythmical extensions; i.e., each current-year (0-year-old) shoot having multiple leaves flushes within a short emergence duration (usually within one month) at the beginning of the growing season. Once a current-year shoot is formed, it usually remains on its mother shoot until autumn when all the leaves and several older annual shoots are shed from the abscission layer formed at their base positions. The number of current-year shoots produced on a mother (1-year-old) shoot tends to increase with the increasing length of the mother shoot. Current-year shoots near the apex of a mother shoot tend to be longer, bear more leaves, and survive more years than those that emerge near the base of the mother shoot due to the lack of strong apical dominance of Konara oaks. Surviving 0 -year-old shoots become 1-year-old shoots the next year and produce new current year shoots that in turn depend on the shoot length and position on the mother shoot. Some 0 -year-old shoots, mostly shorter ones, do not bear new daughter shoots and so die the next year. If we simulate the demography of annual shoots by taking into account such observable patterns, the resulting age structure of annual shoots should be similar to the actual age structure.

Konara oak distributes widely over the warm- and cool-temperate forest zones of Japan, and often becomes the dominant canopy species. From a hardwood forest, branches were randomly sampled from the middle of the top crowns of three Konara trees. The canopy height was about $20 \mathrm{~m}$ and the forest age around 50 years. The age of each annual shoot in the branch sample was determined from bud scales and annual rings on the cross section of the annual shoot. The lengths of each annual shoot for each age, basal position of each annual shoot on its mother shoot, and the number of leaves on each 0-year-old shoot were also recorded. Next, we show an outline of our analyses, using data from one of the branch samples. 


\section{Age structure of a branch}

Initially we examined the age structure of the branch sample. The number of annual shoots for each annual-shoot age was counted, and this was then plotted against the age of annual shoots on semi-log coordinates. The relationship between the number of annual shoots (NA) and the age of the annual shoots (A) could be fairly approximated by the exponential equation (Fig. 1),

$$
\mathrm{NA}=144.3 \exp (-0.782 \mathrm{~A}) \text {, }
$$

where $r^{2}=0.994$ and $p<0.0001$. The value of $\exp (0.782)$ in the equation corresponds to the ratio of the number of annual shoots of a given age divided by that of the one-year older shoots. The value here was 2.19 , showing that the number of annual shoots of a given age was about twice that of the one-year older shoots. As for age structure, it appeared that the number of annual shoots increased exponentially with time, but naturally the age structure is a result of the abscission of older shoots previously present on their mother shoots.

In the next section we introduce a simulation of branch-structure development and show how the observed exponential pattern of age structure can be reconstructed. Some structural patterns observed in a branch are expressed by arbitrary equations approximating the patterns.

\section{Simulation procedure}

For annual shoots older than 2 years, it is possible to estimate the number of 0 -year-old shoots that had been present when their mother shoot was a 1-year-old shoot by observing leaf-scars or shoot-scars. However, the estimation is sometimes difficult because scars become obscure with age. Hence, the length and number of 0-year-old shoots produced on each 1-year-old shoot are the most reliable information obtainable from a branch sample. Therefore, to construct the simulation, only data for 0 - and 1-year-old shoots on the branch sample were used. An underlying assumption in the simulation is that the coefficients of the equations used for the simulation are constant over time, meaning that there is no year-to-year difference in the process of branch development. The four major relationships used for the simulation are: 
1. The relationship between the length of mother shoot $(\mathrm{LM}, \mathrm{cm})$ and the number of daughter shoots on it (ND). It was approximated by

$$
\mathrm{ND}=[(\mathrm{LM}-1.09) / 2.26] \text {, }
$$

where brackets show the Gaussian integer (Fig. 2A). This relationship shows that the number of daughter shoots can be determined simply by mother-shoot length. It should be noted that there is a critical mother-shoot length that does not produce daughter shoots the next year (arrow in Fig 2A). In the present case a mother shoot shorter than $3.35 \mathrm{~cm}$ will not produce any daughter shoots.

2. The relationship between mother-shoot length and the length of the apical daughter shoot (shoot at the apical position on the mother shoot) (LDapex, cm). An apical daughter shoot tends to be the longest of all daughter shoots on a mother shoot. The relationship (Fig. 2B) was approximated by the exponential function,

$$
\text { LDapex=1.25 } \exp (0.224 \mathrm{LM}) \text {. }
$$

As the intersection of this curve and the dotted diagonal line (LDapex=LM) in Fig. 2B shows, the apical-daughter-shoot length was the same as that of mother shoot when the mother-shoot length was $8.6 \mathrm{~cm}$. The length of a mother shoot that bears an apical daughter shoot whose length is the same as that of the mother shoot is referred to as the "recurrent length". The length of an apical daughter shoot was shorter than the mother-shoot length if the mother shoot was shorter than the recurrent length, $8.6 \mathrm{~cm}$.

3. The relationship between the length of the daughter shoot and the position of the daughter shoot on the mother shoot. The daughter-shoot position was expressed by the relative distance from the apex of the mother shoot $(\mathrm{S}, \%$; mother-shoot length $=100 \%$ ), and the daughter-shoot length was represented by its length relative to its mother-shoot length (RL, \%). The relationship (Fig. 2C) was approximated by

$$
\mathrm{RL}=74.6 \mathrm{~S}^{-0.225} \text {. }
$$

Equations (3) and (4) are used in simulations to calculate a daughter-shoot length from a mother-shoot length.

4. The changes in the linear density of 0 -year-old shoots with changes in position on a mother shoot. The daughter shoots tended to be denser near the apex of a mother shoot, and become sparser as the daughter-shoot position approached the base of the mother shoot. This observation was represented by linear density of 0 -year-old shoots $\left(\mathrm{dN} / \mathrm{dS},(10 \%-\mathrm{S})^{-1}\right)$ at $10 \%$ intervals of the relative position on a mother shoot (S, \%) (Fig. 2D). It was approximated by 


$$
\mathrm{dN} / \mathrm{dS}=1.09 \mathrm{~S}^{-1.29} .
$$

If the linear density is integrated from the value corresponding to the position of the apical daughter shoot (defined as $\mathrm{S}=$ Sapex) towards the base of the mother shoot (i.e., $S=100$ ), the integrated value at $S=100$ should correspond to the number of 0 -year-old shoots calculated from a mother-shoot length (Eq. (2)). Based on this principle, we calculated the $\mathrm{S}$ values where the first daughter shoot, the second, the third, etc., appeared on the mother shoot.

The flowchart of the simulation is outlined in Fig. 3. In this simulation the length of a mother shoot determines everything regarding the daughter shoots on it; initially, only a mother shoot with a certain length was given. The length was set to be the recurrent length $(=8.6 \mathrm{~cm}$; see above). This is equivalent to assuming that the annual-shoot length of the main axis of a branch is always the same each year, since a mother shoot with the recurrent length always bears a daughter shoot with the recurrent length at the mother-shoot's apex. The number of daughter shoots on a mother shoot is determined by mother-shoot length by Eq. (2). Then the basal position of each daughter shoot on the mother shoot (Eq. (5)) and the length of each daughter shoot (Eqs. (3) and (4)) are automatically determined. Some of the daughter shoots may not produce new daughter shoots the following year if their lengths are shorter than the critical length for shoot production (Eq. (2)). Such daughter shoots are regarded as being dead the following year. If a twig (a part of the branch composed of several annual shoots) does not bear any 0-year-old shoots, this twig is also regarded as dying (dieback of a twig). Surviving 0-year-old shoots (i.e., those longer than the critical length), become mother shoots next year, each of which produces new daughter shoots the following year according to its length. Thus the developmental process of a branch can be simulated, starting with just one mother shoot in the initial year.

\section{Simulation for branch development}

Figure 4 shows a two-dimensional illustration of the simulated branch structure with eight simulated years. In this illustration, the length of each annual shoot and their positions on their mother shoots are correctly drawn, but the angle of annual-shoot extension is arbitrary. In the first year, five 0-year-old shoots (one apical shoot and four lateral ones) were produced on an initial mother shoot. During the first and third years, 
new 0 -year-old shoots were produced on their respective mother shoots, while the number of 0-year-old shoots was less, and their length shorter, on lateral twigs originating from the lower shoots of the first year. In the fourth year, 0-year-old shoots were not produced on the twig originating from the lowest lateral shoot of the first year, since all the 1-year-old shoots on the twig were shorter than the critical length for shoot production (Fig. 2B). This means that this twig died back to the main stem. By the seventh year, the four twigs whose origins were the four lateral shoots of the first year died out, and only the apical shoot of the first year survived. On the other hand, in the sixth year, the living part of the branch (a part having one or more 0-year-old shoots) was composed of living annual shoots of six years of age. After that, the living part does not change its structure, having been raised upwards with time. This means that a cluster of foliage with a stable structure was formed.

Comparing the structures of the eight simulated years in Fig. 4, we see that the whole first-year structure appears in the upper part of the second-year structure, and the whole second-year structure in the upper part of the third-year structure, and so on. Hence we could say that the developmental process of the stable cluster is as if less-developed lateral twigs are added to their lower part each year until a stable cluster is formed.

6 Simulated increase in the number of current-year shoots and age structure

As the formation of the stable cluster suggests, the total number of current-year shoots reaches a constant value in the sixth year (Fig. 5, dotted line). In the seventh year, the number of 0-year-old shoots developing on the four lateral shoots of the first year died out, meaning that the four lateral twigs died back, while the number of 0-year-old shoots developing on the apical shoot of the first year reached a constant. This indicates that the turnover time of the living part of the cluster structure is the same as the time it takes for all the lateral shoots produced in the first year to die out (six years in this case). An exponential age structure of annual shoots as in Fig. 1 was also reproduced with the simulation. Figure 6 shows the age structure of living annual shoots in the seventh year of the simulation. Note that the exponential relationship holds for the annual shoots from zero to six years of age, which corresponds to the turnover time of the cluster. This explains why branches with an exponentially forking structure can exist in a crown with 
little space for increasing its size; the exponential relationship only holds in the stable cluster that does not increase in size over time. For annual shoots over six years of age, the number of survived shoots is just one (Fig. 6), which had been the apical shoot of the first year.

7 Ecological implications of the formation of a stable cluster

The data used for the present simulation was for a branch sample taken in the middle of a crown. Hence the simulated stable cluster should be a reflection of intra-crown (i.e., inter-shoot) competition rather than inter-crown (neighborhood) competition. In forming a stable cluster, dieback of older lateral twigs would be an important process, since it suppresses the exponential increase of current-year shoots. The physiological mechanisms that cause dieback of lateral twigs are unknown, but they would be strongly affected by physical and biological conditions. If the relationships in Figs. 1 and 2 vary according to ambient physical (air temperature and humidity, light intensity, light quality, etc.) and biological (inter-shoot competition, etc) conditions where the branch exists, the size and turnover time of the simulated stable cluster would also vary. Once a forest canopy is closed, tree crowns need to exist with little space to spread foliage, while they have a natural characteristic that multiple current-year shoots are produced on each mother shoot when they flush new leaves. Formation of a stable cluster of branches can partly explain how such tree crowns can continue to exist in a closed canopy. In fact, if we can look down on a forest from above, we can observe that a crown of a hardwood tree is composed of clusters of foliage, which can be regarded as basic components of a hardwood crown (e.g., Kira et al. 1969). Persistence of hardwood crowns in a closed canopy may be closely related to the ability to form stable clusters of foliage.

Acknowledgment. We thank Tomohiro Mano, Asako Togashi, Chika Kanada and other students of the laboratory of Forest Ecology, Gifu University, for their assistance with fieldwork, and Mutsuki Higo, Shogo Kato, Akira Komiyama, Toshihiko Hara and Shin-Ichi Yamamoto for facilitating the study. This research was partly supported by the Ministry of Education, Science, Sports and Culture, Japan, through a Grant-in-Aid for Scientific Research (A2) (No 13356003 , Yamamoto S). 


\section{References}

Buck-Sorlin GH, Bell AD (2000) Crown architecture in Quercus petraea and Q. robur: the fate of buds and shoots in relation to age, position and environmental perturbation. Forestry $73: 331-349$

Chaar H, Colin F, Collet C (1997) Effects of environmental factors on the shoot development of Quercus petraea seedlings. A methodological approach. For Ecol Manage 97: 119-131

Collet C, Frochot H (1996) Effects of interspecific competition on periodic shoot elongation in oak seedlings. Can J For Res 26: 1934-1942

Kira T, Shinozaki K, Hozumi K (1969) Structure of forest canopies as related to their primary productivity. Plant Cell Physiol 10: 129-142

Koike F (1989) Foliage-crown development and interaction in Quercus gilva and $Q$. acuta. J Ecol 77: 92-111

Sattler R, Rutishauser R (1997) The fundamental relevance of morphology and morphogenesis to plant research. Ann Bot 80: 571-582

Steingraeber DA, Kascht LJ, Franck DH (1979) Variation of shoot morphology and bifurcation ratio in sugar maple (Acer saccharum) saplings. Amer J Bot 66: $441-445$

Sumida A, Terazawa I, Togashi A, Komiyama A (2002) Spatial arrangement of branches in relation to slope and neighbourhood competition. Ann Bot 89: 301-310

Suzuki A (2000) Patterns of vegetative growth and reproduction in relation to branch orders: the plant as a spatially structured population. Trees 14: 329-333

Suzuki M (2001) Allometry and dynamics of current-year shoot populations in the crown development of deciduous trees. Ph.D. Thesis, Graduate School of Earth Environmental Science, Hokkaido University

Seleznyova AN, Thorp TG, Barnett AM, Costes E (2002) Quantitative analysis of shoot development and branching patterns in Actinidia. Ann Bot 89: 471-482

White J (1979) The plant as a metapopulation. Ann Rev Ecol Syst 10: 109-145

Wilson BF (1989) Tree branches as population of twigs. Can J Bot 67: 434-442 


\section{Figure Legends}

Fig. 1. Age structure observed for a branch sample of Quercus serrata as expressed by an exponential relationship between the age of annual shoots and the number of annual shoots.

Fig. 2A-D. Observed structural patterns of annual shoots used in the simulation. Explanation in text.

Fig. 3. Flowchart of the simulation.

Fig. 4. Branch structure development over eight simulated years. Thick solid lines show 0 -year-old shoots. Lateral twigs developing from the four lateral 0 -year-old shoots of the first year are shown by thick gray lines.

Fig. 5. Simulated increase in the number of 0 -year-old shoots with time. The number of 0 -year-old shoots on the twigs originating from the apical shoot and the four lateral shoots of the first simulated year are shown by thick lines.

Fig. 6. Simulated relationship between the age of annual shoots $(A)$ and the number of annual shoots $(N A)$. The relationships was approximated by $N A=79.7 \exp (-0.684 A)$ $\left(r^{2}=0.996, p<0.001\right)$. The slope was not significantly different from the relationship in Fig. $1(p>0.05)$. 


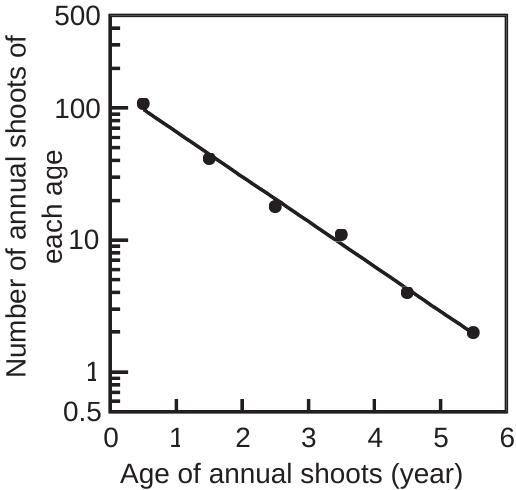


Initial length of the original mother shoot, LM

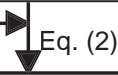

Number of daughter shoots on a mother shoot, ND

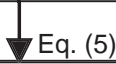

Position of each daughter shoot on a mother shoot, S

Eqs. (3), (4)

Length of each daughter shoot, LM $\times \mathrm{RL}$

Critical length (Eq. (2))

Survived daughter shoots

(mother shoots next year) 


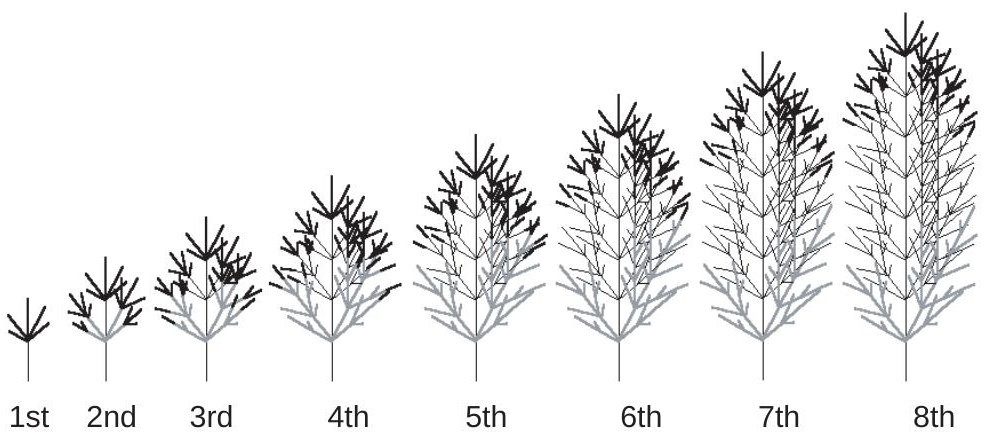




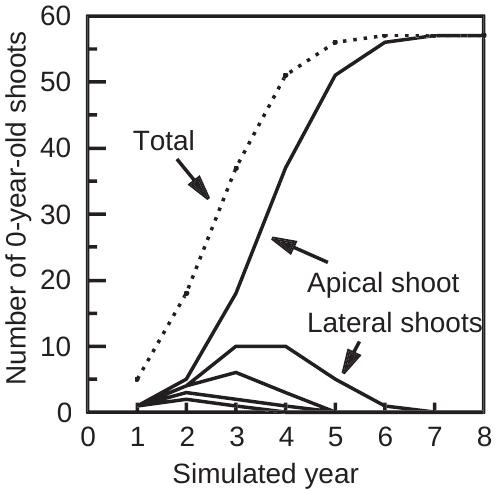




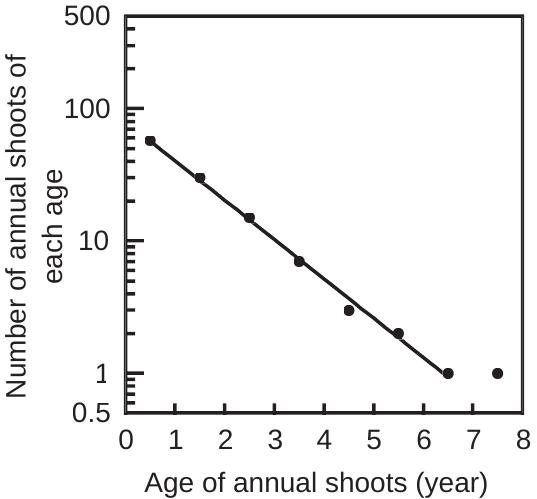

